\title{
Parálisis facial permanente: corrección quirúrgica con la técnica de Labbé
}

\author{
Permanent facial paralysis: surgical correction with Labbe's technique
}

\author{
Rubén Yangali ${ }^{1}$, Alex Neme ${ }^{2}$, Kattia Moreno ${ }^{3}$, Jerson Cuadros ${ }^{4}$ \\ ${ }^{1}$ Cirujano de Cabeza Cuello y Maxilofacial, Servicio de Otorrinolaringología y Cirugía de Cabeza y Cuello. Hospital Central de la Fuerza Aérea del Perú. Lima, Perú. \\ ${ }^{2}$ Cirujano de Cabeza Cuello y Maxilofacial. Servicio de Urgencias. Hospital La Mancha Centro. Ciudad Real, España. \\ ${ }^{3}$ Cirujano de Cabeza Cuello y Maxilofacial. Research Assistant Otolaryngology and Head and Neck Surgery. University Of Cincinnati. Ohio, USA. \\ ${ }^{4}$ Licenciado en Terapia de Lenguaje. Centro de Terapia Física y Rehabilitación (CETEFI). Arequipa, Perú
}

\section{Resumen}

La parálisis facial genera un gran déficit estético y funcional. Las diversas técnicas quirúrgicas han ido evolucionando, a fin de corregir este defecto de la forma más estructural y fisiológica posible. Consideramos que la mioplastia de alargamiento del temporal constituye actualmente la técnica de elección para corregir los casos de parálisis facial permanente.

Palabras clave: Parálisis facial; nervio facial; cirugía; músculo temporal.

\begin{abstract}
Facial paralysis causes a large aesthetic and functional defect. Different surgical techniques may be used to repair this defect. Some of them have focused in structural and physiological aspects. We consider that temporalis lengthening myoplasty is currently the most impor tant technique for permanent facial paralysis repair.
\end{abstract}

Key words: Facial paralysis; facial nerve; surgery; temporal muscle.

\section{INTRODUCCIÓN}

La parálisis facial permanente es siempre una entidad mal tolerada por la persona afectada. Los problemas tanto estéticos como funcionales que esta produce, generan siempre gran frustración en el paciente. La asimetría facial, la dificultad para hablar, la incontinencia salival, la epífora y la afectación corneal en el ojo del lado afectado son algunas de las manifestaciones más características.

Esta entidad es producida por lesión del nervio facial (VII par craneal), sea a nivel de sus núcleos formadores en el tronco cerebral o a cualquier nivel de su trayecto. Dicho trayecto se inicia en el bulbo raquídeo, pasa por la base del cráneo y termina en diversas ramas que atraviesan la parótida, para finalmente inervar los músculos faciales. Es por ello que, dependiendo del nivel de la injuria, podemos tener parálisis faciales totales o parciales. Entre las causas más frecuentes de injuria al nervio facial se encuentran los tumores, los procedimientos quirúrgicos y las causas idiopáticas ${ }^{(1-4)}$.

Muchos procedimientos quirúrgicos han sido ideados con el fin de mejorar

las secuelas producidas por una parálisis facial permanente, técnicas quirúrgicas que van desde simples tracciones, con el fin de equilibrar la asimetría facial; son conocidas también como técnicas estáticas (interposición y tracción con fascia autóloga, hilos rusos, entre otros) ${ }^{(5,6)}$ (figura 1). Y otras que pretenden dotar de cierta movilidad al lado afectado, como anastomosis hipoglosofacial, colgajos fasciomusculares y recientemente técnicas más complejas, que buscan dar movilidad voluntaria al lado afectado, consiguiéndose incluso sonrisas

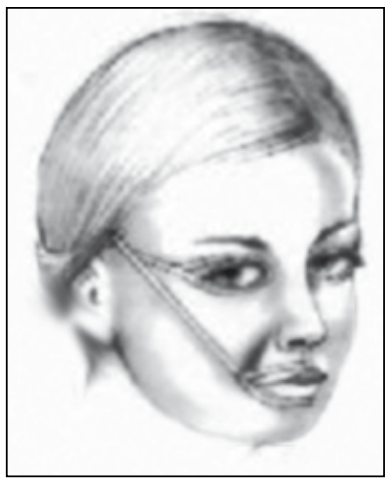

Figura 1. Técnica estática. espontáneas, conocidas como técnicas dinámicas ${ }^{(7-10)}$.

La mioplastia de alargamiento del músculo temporal, técnica dinámica e innovadora, dada a conocer por Labbé en esta década, constituye actualmente una de las mejores técnicas para el tratamiento de la parálisis facial permanente, ya que no solo mejora la simetría facial, sino que además mejora la incontinencia salival, el tono facial, mejora el habla, dota al paciente de movimiento en el lado afectado, así como la capacidad de volver a sonreír. Evocando antiguas técnicas, como la de Gilles (1934) (7) (figura 2) y la de Mac Laughlin (1953) ${ }^{(10)}$, las perfecciona, ya que no transpone solo un haz de fibras fascio-musculares del músculo temporal, ni injerta fascia autóloga, sino que transpone la totalidad del músculo temporal hacia un nuevo punto de inserción en la musculatura de la comisura labial, dotándola de una verdadera fuerza contráctil y por tanto de movimiento ${ }^{(8)}$.

Hacemos una breve revisión de la técnica de Labbé, aplicada en un paciente con parálisis facial permanente unilateral. 


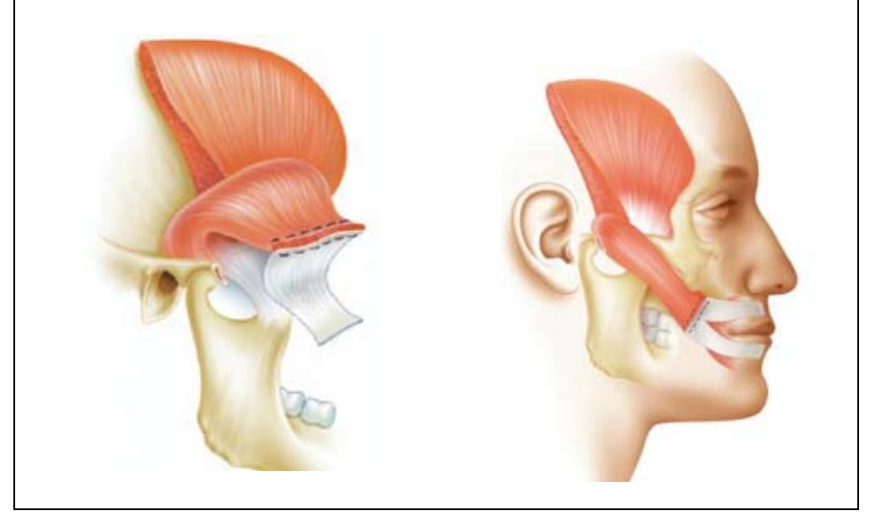

Figura 2. Técnica de Gilles.

\section{EVALUACIÓN PREOPERATORIA}

La evaluación preoperatoria debe incluir el estudio del tipo de sonrisa del paciente. Para ello, se hace la comparación respectiva con el lado sano y se utiliza fotos anteriores a la parálisis. Los tipos de sonrisa, según la clasificación de Rubín, pueden ser: de Mona lisa, tipo canina y por último la tipo completa o full teeth ${ }^{(11-14)}$ (figura 3).

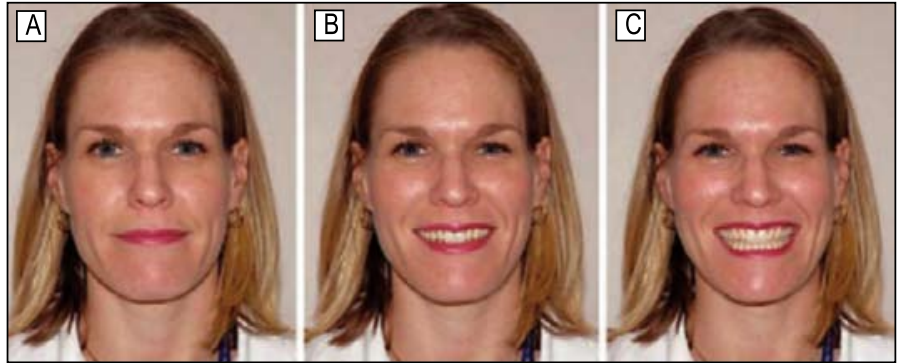

Figura 3. Tipos de sonrisa. A: Tipo de Mona Lisa. La sonrisa involucra primariamente la elevación de la comisura. B: Tipo canina. La sonrisa adiciona la contracción del elevador del labio. C: Tipo completa o full teeth, que incluye la contracción del depresor del ángulo de la boca ${ }^{(14)}$.
Con el paciente en decúbito dorsal, y bajo anestesia general e intubación orotraqueal (el tubo orotraqueal debe ir fijado con seda a la línea media en los incisivos superiores y no con cinta adhesiva, a fin de dejar libre la zona de las comisuras labiales y los surcos nasogenianos), se realiza una incisión coronal, incidiendo con el bisturí en angulación a fin de evitar en lo posible áreas de alopecia futura. La incisión va desde el nivel del trago en el área preauricular del lado afectado hasta el área supraauricular, en el lado sano; se eleva el colgajo subgaleal, exponiéndose la fascia temporal que trasluce el músculo. A $2 \mathrm{~cm}$ por encima del reborde orbitario, se incide la fascia pericraneana y la fascia temporal superficial y se las eleva, a fin de esqueletizar el reborde orbitario, el cuerpo del malar, el arco cigomático y las fibras dístales del músculo temporal. Expuestas estas estructuras, se procede, con la ayuda de una sierra alternante, a la sección anterior y posterior del arco cigomático, el cual queda unido a las fibras del masetero; en este momento, se verifica que un delgado haz de fibras musculares del masetero se inserte también en el temporal. Estas fibras deben ser seccionadas, a fin de exponer el tendón terminal del temporal en su inserción en la apófisis coronoides, estructuras que están sumergidas en la extensión adiposa de la bola de bichat. Se procede luego a seccionar la apófisis coronoides, con el tendón del temporal unido a ella. Luego, se pasa a incidir la fascia y el músculo
La clasificación del tipo de sonrisa determinará, en el intraoperatorio, áreas preferentes de fijación del tendón temporal; de ahí su importancia. Los estudios electromiográficos y arteriogramas están indicados para evaluar la integridad del músculo temporal ${ }^{(8,9)}$.

\section{TÉCNICA QUIRÚRGICA}

El aspecto conceptual más importante de la técnica de Labbé radica en que transpone toda la función y fuerza de contracción del músculo temporal (de su inserción natural en la apófisis coronoides mandibular) hacia la musculatura de la región de la comisura labial ${ }^{(8,9)}$. Figura 4.

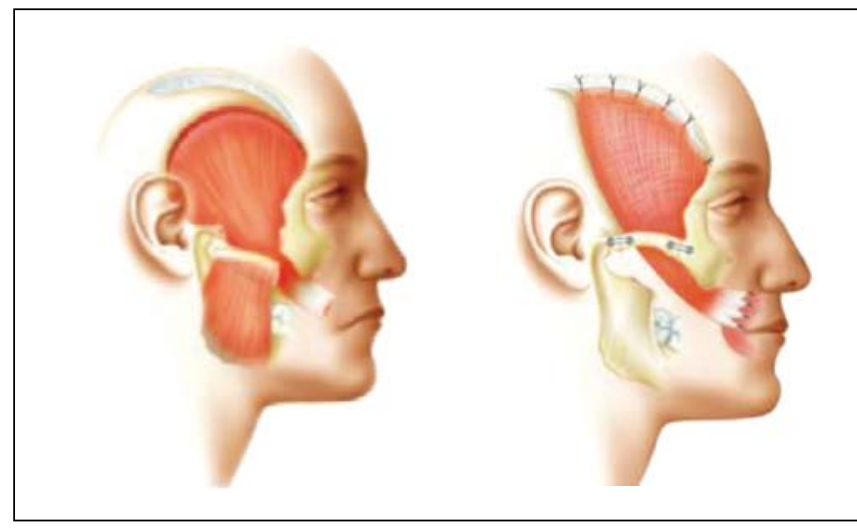

Figura 4. Imagen que resume la técnica quirúrgica, descrita por Labbé. El temporal desciende y avanza en su totalidad y es fijado a la musculatura peribucal. 
temporal, de su inserción en el cráneo, dejando $1 \mathrm{~cm}$ de fascia unida al cráneo (para la posterior recolocación y sutura del temporal). Se procede a desinsertar y elevar todo el músculo temporal del cráneo, llegando a la línea ósea esfenotemporal, teniendo especial cuidado con la disección, a fin de no dañar el complejo neurovascular que inerva e irriga dicho músculo. Luego, se procede a incidir a nivel del surco nasogeniano, disecándose el músculo orbicular; entonces, se toma con una pinza la apófisis coronoides con el tendón temporal unido a ella y se les tuneliza a través de la bola de bichat y por debajo del malar, emergiendo así dichas estructuras por nuestra incisión

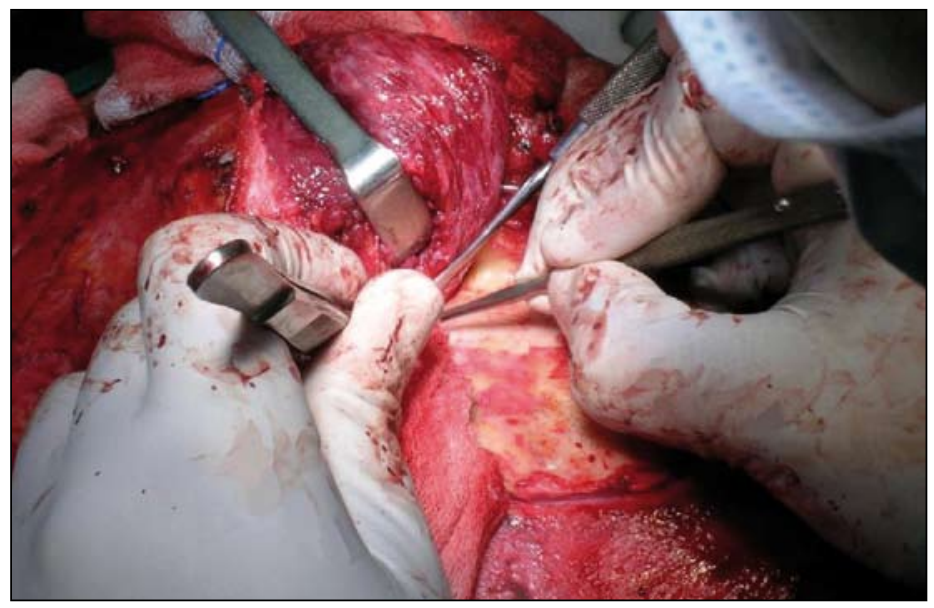

Figura 5. Imagen intraoperatoria, que muestra la separación completa del músculo temporal del cráneo y la aproximación a la línea esfenomaxilar. nasogeniana; haciendo esta maniobra, se desciende y avanza el temporal de su posición natural. Se retira el coronoides del tendón temporal, se extiende el tendón y se sutura con prolene $3 / 0$ a la musculatura peribucal, desde el nivel de la comisura hasta el ala nasal. Una ligera sobrecorrección es importante para mejores resultados. Por último, se fija el arco cigomático con miniplacas y tornillos de titanio, se deja drenajes aspirativos, se sutura por planos de manera habitual; la incisión nasogeniana se sutura con nylon 6/0. Suelen ser necesarias miomectomías en el lado sano, a fin de mejorar áreas de hipercontracción muscular ${ }^{(4,8,9)}$. Figuras 5 y 6.

\section{CIERRE PALPEBRAL}

La incapacidad para un buen cierre palpebral y el ectropión en los pacientes con parálisis facial permanente condicionan epífora y daño corneal progresivo; de ahí la importancia de su manejo.

La tarsorrafia asimétrica con interposición de fascia en el elevador del párpado, la suspensión del párpado inferior, la colocación de pesas de oro en el párpado superior, entre otras, son algunas de las técnicas que son individualizadas según la severidad del cuadro del paciente, consiguiendo así una buena protección ocular $^{(2,3,15)}$ (figura 7).

De esta manera, se complementa el manejo quirúrgico integral del paciente con parálisis facial permanente. (Ver video).

\section{DISCUSIÓN}

Es muy importante destacar que, ante cualquier injuria del nervio facial, la primera opción siempre será la reparación inmediata del nervio; cuando esto no ocurre, la parálisis facial será inevitable. En los casos en que se realiza la reparación del nervio y en los casos idiopáticos, el paciente debe entrar a un protocolo de rehabilitación; se debe esperar por lo menos un año para la respuesta a este manejo; vencido este plazo, recién se puede considerar las opciones quirúrgicas. $(1,2,6,6)$

Las diferentes técnicas quirúrgicas diseñadas para el manejo de la parálisis facial

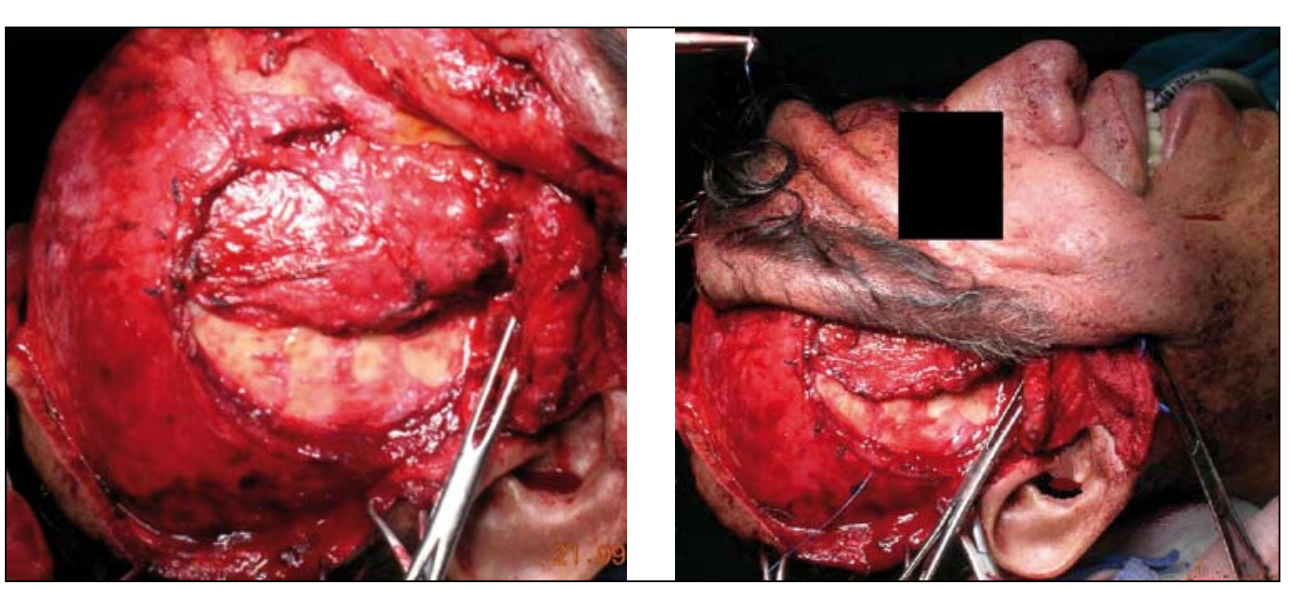

permanente han evolucionado. Las técnicas estáticas, que solo buscan equilibrar la asimetría facial a través de tracciones, están siendo desplazadas por técnicas dinámicas de cada vez mayor complejidad y que buscan, además, dotar de movimiento al lado afectado $(4,8,9,14)$.

Las anastomosis hipoglosofacial, si bien es cierto logran resultados satisfactorios al mejorar el tono muscular afectado, difícilmente logran movimiento en el lado afec-
Figura 6. Imágenes intraoperatorias, que muestran el reposicionamiento del temporal adelantado y descendido, respecto a su posición original y la ligera sobrecorrección que se ejerce sobre la musculatura peribucal. 


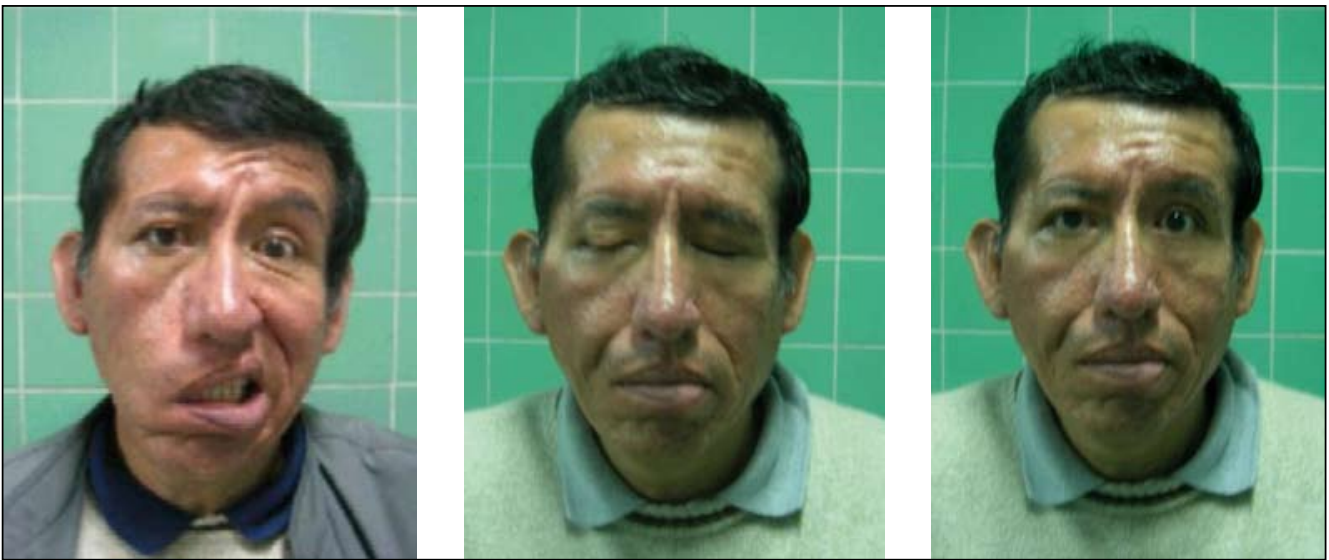

Figura 7. Paciente con parálisis facial post tumor del ángulo pontocereboloso. El cierre palpebral fue corregido con una pesa de oro. La parálisis facial fue corregida con la técnica de Labbé. Obsérvese la simetría facial a 6 meses poscirugía.

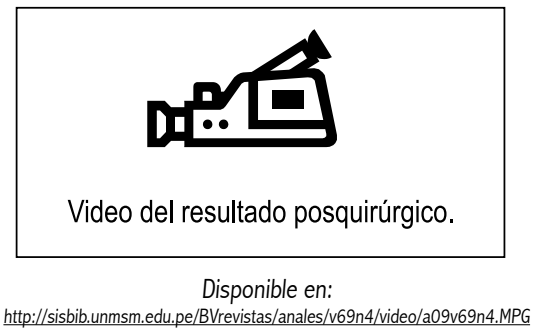

tado y tienen el inconveniente que pueden generar hipotrofia de la mitad de la lengua ${ }^{(17)}$.

Las primeras técnicas con fines dinámicos, que también buscan movilidad a través de la contracción del músculo temporal, tienen el inconveniente de que buscan dicho movimiento contráctil a través de una doble función muscular, como en el caso de la técnica de Gilles o de la interposición de fascia autóloga al verdadero punto de tracción, como en la técnica de Mac Laughlin, por lo que los resultados no son del todo satisfactorios. $(2,7,10)$.

La técnica de Labbé - llamada mioplastia de alargamiento del músculo temporal- mejora antiguas técnicas con fines dinámicos, ya que transfiere directamente un punto móvil de la apófisis coronoides a otro punto móvil en la musculatura peribucal, sin mayor alteración en el punto base de tracción a nivel del hueso temporal; esto condiciona que toda la fuerza contráctil del músculo temporal se dirija solo a movilizar la musculatura peribucal, obteniéndose por ello mejores resultados.

La terapia física de rehabilitación posquirúrgica es capital para obtener resultados satisfactorios. Estas deben iniciarse precozmente, alrededor de la segunda semana del postoperatorio. Inicialmente, se reeduca y potencia al temporal en su función primaria. De esta manera, se logra en una primera etapa una sonrisa llamada sonrisa mandibular, es decir, aquella que se logra con el apretado de dientes; posteriormente, se obtiene la independencia contráctil del temporal de los movimientos mandibulares, consiguiéndose la llamada sonrisa temporal; y, por último, puede lograrse, con protocolos de terapia de alrededor de 6 a 18 meses, la llamada sonrisa espontánea, que es aquella gesticulación de risa voluntaria y espontánea que acontece ante un evento risible. Por ello, es importante concientizar al paciente de la importancia de seguir con el protocolo de rehabilitación, ya que buena parte del éxito dependerá de su entusiasmo y perseverancia ${ }^{(6,16,18,19)}$.

Se concluye que la mioplastía de alargamiento del músculo temporal, descrita por Labbé, constituye actualmente una de las mejores técnicas quirúrgicas con fines dinámicos, para el manejo de pacientes con parálisis facial permanente; su cierta mayor complejidad quirúrgica, respecto a técnicas anteriores, se compensa largamente por los mejores resultados obtenidos y la satisfacción de los pacientes. Esta técnica requiere ser complementada con técnicas que aborden la protección ocular, para así dar un manejo quirúrgico integral al paciente con parálisis facial permanente.

La terapia de rehabilitación física influye sobre el resultado final. Concientizar al paciente de su importancia, a fin de que concluya con el protocolo de rehabilitación, es vital para la obtención de mejores resultados.

\section{REFERENCIAS BIBLIOGRÁFICAS}

1. Bascom DA, Schaitkin BM, May M, Klein S. Facial nerve repair: a retrospective review. Facial Plast Surg. 2000;16(4):309-13.

2. Fernández J, Salmerón J, Calderón J y col. Rehabilitación de la parálisis facial mediante el colgajo del músculo temporal y los implantes de laminas de oro. Acta Otorrinolaring Esp. 1999;50(1):20-8.

3. Keen M. Gold eyelid weights in patients with facial palsy. Plast Reconstr Surg. 1992;90(6):11212.

4. Campos 0, Gutiérrez T. Parálisis facial permanente: tratamiento quirúrgico en base a la técnica de Labbé. Rev Chil Cir. 2006;58:159-64.

5. Ragnell A. Experience with dynamic and static reconstruction in cases of facial paralysis. Scand J Plast Reconstr Surg. 1968;41:343.

6. Alam D. Rehabilitation of long-standing facial nerve paralysis with percutaneous suture-based slings. Arch Facial Plast Surg. 2007;9:205-9. 
7. Gillies H. Experiences with fascia lata grafts in the operative treatment of facial paralysis. Proc R Soc Med. 1934;27:1372-9.

8. Labbé D, Huaut M. Lengthening temporalis myoplasty and lip reanimation. Plast Reconstr Surg. 2000;105(4):1289-97.

9. Labbé D, Bardot, J. Técnicas quirúrgicas. Parálisis facial periférica [CD-ROM]. Paris: Éditions Scientifiques et Médicales Elsevier SAS; 2001.

10. Mc Laughlin CR. Surgical support in permanent facial paralysis. Plast Reconstr Surg. 1953;11:302-14.

11. Rubin LR. The anatomy of a smile: Its importance in the treatment of facial paralysis. Plast Reconstr Surg. 1974;53(4):384-7.

12. Rubin LR, Mishriki Y, Lee G. Anatomy of the nasolabial fold: the keystone of the smiling mechanism. Plast Reconstr Surg. 1989;83:1-8.

13. Rubin LR. The anatomy of smile: Its importance in the treatment of facial paralysis. Plast Reconstr Surg. 1989;83:1-8.
14. Byrne PJ, Kim M, Boahene K, Millar J, Moe $K$. Temporalis tendon transfer as part of a comprehensive approach to facial reanimation. Arch Facial Plast Surg. 2007;9:234-41.

15. Krastinova-Lolov D. Chirurgie palliative des secuelles de paralysie fasciale péripherique. Com SOC FCPRE. Paris, Francia. 1989.

16. Diels HJ. Facial paralysis: Is there a role for the therapist? Facial Plast Surg. 2000;16(4):3614.

17. López D, López D, Campos ME. La anastomosis hipogloso-facial como solución de una parálisis facial residual completa. Nuestra experiencia. Acta Otorrinolaringol Esp. 2006;57:223-7.

18. Lambert-Prou MP. Prise en charge orthophonique de la paralysie faciale périphérique corrigeé par transfert du muscle temporal sur la commissure labiale. Revue Glossa. 1998;63:4-25.

19. Lambert-Prou MP. Le sourire envisage. Réponses orthophoniques et chirurgicales a la paralysie faciale périphérique. Paris: L'Ortho Édition; 2003.

Manuscrito recibido el 28 de noviembre de 2008 y aceptado para publicación el 22 de diciembre de 2008.

Correspondencia:

Dr. Rubén Yangali López

Servicio de Otorrinolaringología y Cirugía de

Cabeza y Cuello. Hospital Central de la

Fuerza Aérea del Perú (FAP)

Av. Aramburu 200, Miraflores

Lima 18, Perú

Correo-e: dr.yangali@yahoo.com bengali_r@yahoo.com 